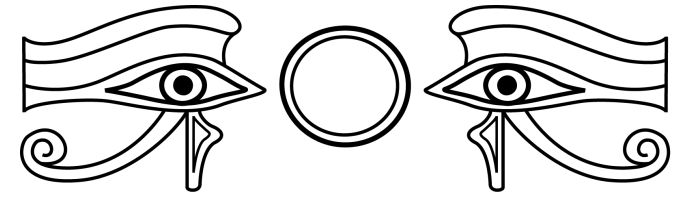 \\ SOCIÉTÉ D’ÉGYPTOLOGIE \\ GENÈVE
}

BULLETIN N 32

\section{Quelques remarques sur un porteur de palanche figuré dans le mastaba de Néfer et Kahai}

\section{Simon DeLVAuX}

Chercheur indépendant

simon.delvaux@wanadoo.fr

orcid : 0000-0002-6336-3728

\section{Résumé}

Comment la représentation d'un porteur de palanche dans un mastaba conduit à s'interroger sur la perception que nous avons des conventions artistiques égyptiennes.

Mots-clés : porteur ; palanche ; mouvement ; posture ; iconographie
Allée de Bon Accueil 64

Bât La Bruyère, apt 90

F-34090 Montpellier (France)

\begin{abstract}
How the representation of a yoke-bearer in a mastaba leads to questioning the perception we have of Egyptian artistic conventions.
\end{abstract}

Keywords: bearer; yoke; movement; posture; iconography

\section{Comment citer/How to cite}

Simon DelvauX, «Quelques remarques sur un porteur de palanche figuré dans le mastaba de Néfer et Kahai », BSÉG 32 (2021), pp. 55-68. 


\title{
Quelques remarques sur un porteur de palanche figuré dans le mastaba de Néfer et Kahai
}

\author{
Simon DelVAUX
}

How the representation of a yoke-bearer in a mastaba leads to questioning the perception we have of Egyptian artistic conventions.

Les porteurs de palanches, personnages fréquemment figurés dans les tombes et mastabas, présentent une grande variété de postures. Pour l'Ancien Empire, on dénombre quinze types de postures « statiques » auxquelles il faut en ajouter trois dans lesquelles le personnage est en mouvement. Parmi ces dernières, celle d'un porteur conservé sur un bas-relief de la paroi est de la chapelle du mastaba de Néfer et Kahai ${ }^{1}$ (Saqqâra Nord, $V^{\mathrm{e}}$ dynastie), comporte deux innovations qu'il convient de remarquer. Enfin, ajoutons qu'il sera à de nombreuses reprises dans cet article fait recours à des vues créées de toute pièce par l'auteur à partir de la vue originelle. Il est donc demandé au lecteur de conserver à l'esprit que ces illustrations ne sont là que pour expliciter le propos. Ces vues inventées seront signalées par la légende : (création de l'auteur).

\section{Les postures du porteur de palanche}

Parmi les quatre-vingt-dix figurations de porteurs de palanche recensées pour l'Ancien Empire, deux grands types de postures peuvent déjà être isolés, les postures « statiques » (tableau 1) et les postures en mouvement (tableau 2$)^{2}$.

${ }^{1}$ A.M. Moussa, H. Altenmüller, The Tomb of Nefer and Ka-Hay (ArchVer 5), Mayence 1971, pp. 21-23, pl. 5 ; M. LASHIEN, The Chapel of Kahai and his Family, (ACE Reports 33), Oxford 2013, pp. 30-31, pl. 16, 81-82.

${ }^{2}$ Cette typologie a été établie sur la base de 90 exemples répertoriés pour cette époque. S. Delvaux, Etudes sur les modes de transport terrestre en Égypte de l'Ancien au Nouvel Empire, Volume II, Thèse de doctorat en égyptologie, sous la direction de Bernard Mathieu, Montpellier, Université Paul Valéry, 2016, pp. 6-33. 


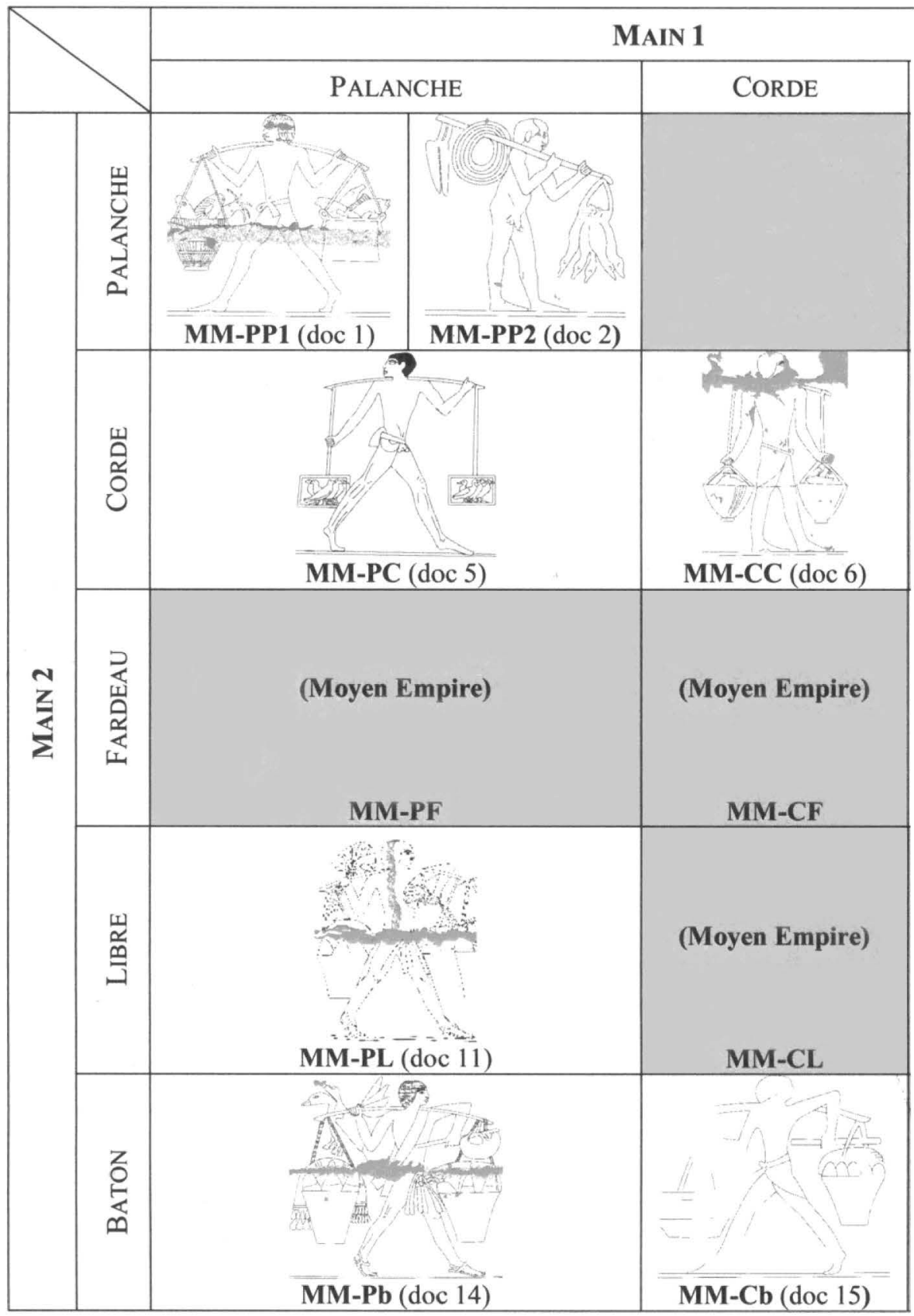

Tableau 1 : les postures «statiques » 


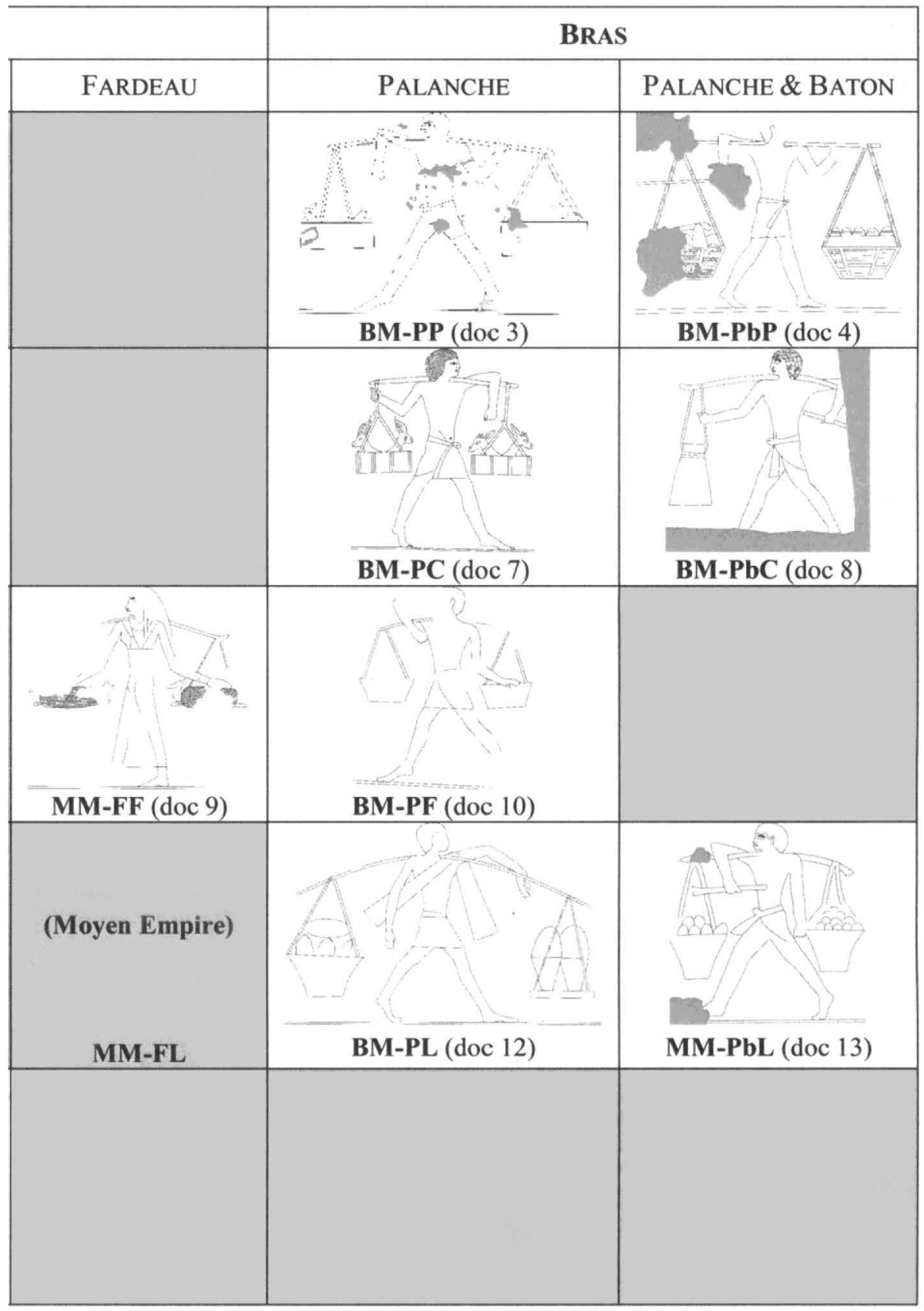




\subsection{Les postures «statiques »}

Les postures dites «statiques » sont ainsi nommées par rapport à la palanche et non au porteur. Ce dernier peut être en train de marcher, par contre sa palanche est dans une position figée à cet instant-là et aucun déplacement prochain de celleci n'est annoncé. Toutefois il ne faut pas imaginer ces postures comme étant définitives. Le porteur peut adapter le positionnement de sa palanche durant son trajet pour répondre à un besoin particulier comme faire cesser un mouvement de balancier qu'aurait pris la charge lors de la marche, rééquilibrer la palanche ou bien soulager une douleur causée par son port.

La typologie exposée dans le tableau 1 se base sur quatre-vingt occurrences de figurations de porteurs de palanche. Elle s'appuie d'abord sur l'usage que le porteur fait de ses mains (type MM) ou bien de son bras et de sa main (type BM), puis sur ce qu'il saisit pour maintenir l'équilibre de sa palanche. Sont notés dans le tableau, pour indication, les types de postures apparaissant au Moyen Empire.

De tous ces types, celui le plus fréquemment représenté est MM-PP1 (trente-six occurrences) et c'est d'ailleurs cette posture qui est utilisée pour figurer le hiéroglyphe A344 8.7\% 8 . Le second type le plus représenté est BM-PP (douze occurrences), qui est en quelque sorte une variation de MM-PP, le porteur tenant là aussi sa palanche à l'aide de ses deux membres supérieurs dans une attitude toutefois plus nonchalante.

\subsection{Les postures en mouvement}

Avec seulement dix occurrences recensées pour l'Ancien Empire, les postures en mouvement sont bien moins représentées que les postures dites «statiques». Plusieurs de ces exemples présentant des lacunes, il est difficile, voire impossible, d'en réaliser la typologie. Toutefois, sans trop pousser l'analyse, on peut déjà avancer que ces postures correspondent à trois moments distincts du transport.

\begin{tabular}{|l|}
\hline \multicolumn{1}{|c|}{ Mouv-1 (doc 16) } \\
\hline $\begin{array}{l}\text { Ce type de posture s'observe dans les moments } \\
\text { précédant le départ. Il correspond au } \\
\text { chargement de la palanche et aux manœuvres } \\
\text { inhérentes. L'un des fardeaux est au sol ou est } \\
\text { en train d'être levé. Le regard du porteur se } \\
\text { pose sur ce dernier. }\end{array}$
\end{tabular}




\begin{tabular}{|l|l|}
\hline \multicolumn{1}{|c|}{ Mouv-2 (doc 17) } \\
\hline $\begin{array}{l}\text { Ce type de posture s'observe dans les moments } \\
\text { où le porteur est sur le départ (ses deux pieds } \\
\text { sont posés au sol) ou en train de partir (l'un de } \\
\text { ses pieds est levé). L'un de ses coudes pointe } \\
\text { vers le ciel, ce qui témoigne qu'il est en train } \\
\text { de finir d'installer la palanche sur ses épaules. }\end{array}$ \\
\hline $\begin{array}{l}\text { Ce type de posture s'observe à l'arrivée à } \\
\text { destination du porteur et au déchargement de sa } \\
\text { cargaison. Pour ce faire il peut, debout, se } \\
\text { contenter de retourner l'un après l'autre ses } \\
\text { fardeaux ou bien retourner un fardeau tout en } \\
\text { veillant à l'équilibre de l'autre. Autrement, } \\
\text { penché en avant, il pose ses fardeaux au sol } \\
\text { pour les vider. }\end{array}$ \\
\hline
\end{tabular}

Tableau 2 : Les postures en mouvement

\section{Le porteur de palanche du mastaba de Néfer et Kahai}

La scène qui nous intéresse est conservée sur la paroi est de la chapelle du mastaba de Néfer et Kahai (Saqqâra nord) et plus particulièrement dans le troisième registre en partant du haut. Sur ce dernier figurent, de gauche à droite :

a. Des hommes en train de cuire des pains. Représentés les épaules rabattues, ils sont vus de profil.

b. Un homme en train de charger des oiseaux dans une cage suspendue à une palanche portée par un autre homme.

c. Un second porteur de palanche qui, il faut le noter, est dans une position rarement figurée. Il s'agit en l'occurrence d'une posture de type MM-PP2, c'est d'ailleurs ce porteur qui est représenté dans le tableau 1 dans l'entrée correspondante.

d. Enfin à droite se trouve un filet dans lequel sont enfermés des oiseaux.

Notre étude s'intéressera au groupe de deux hommes figurés en b et plus particulièrement au porteur de palanche.

\subsection{Description de la posture}

Dans cette scène (fig. 1), le porteur a le bras gauche posé sur sa palanche qu'il tient de la main droite. De part et d'autre, des caisses sont suspendues. À gauche 
un homme est en train de mettre des oiseaux dans l'une d'elles. La posture représentée appartient au type «MOUV- $1 »^{3}$.

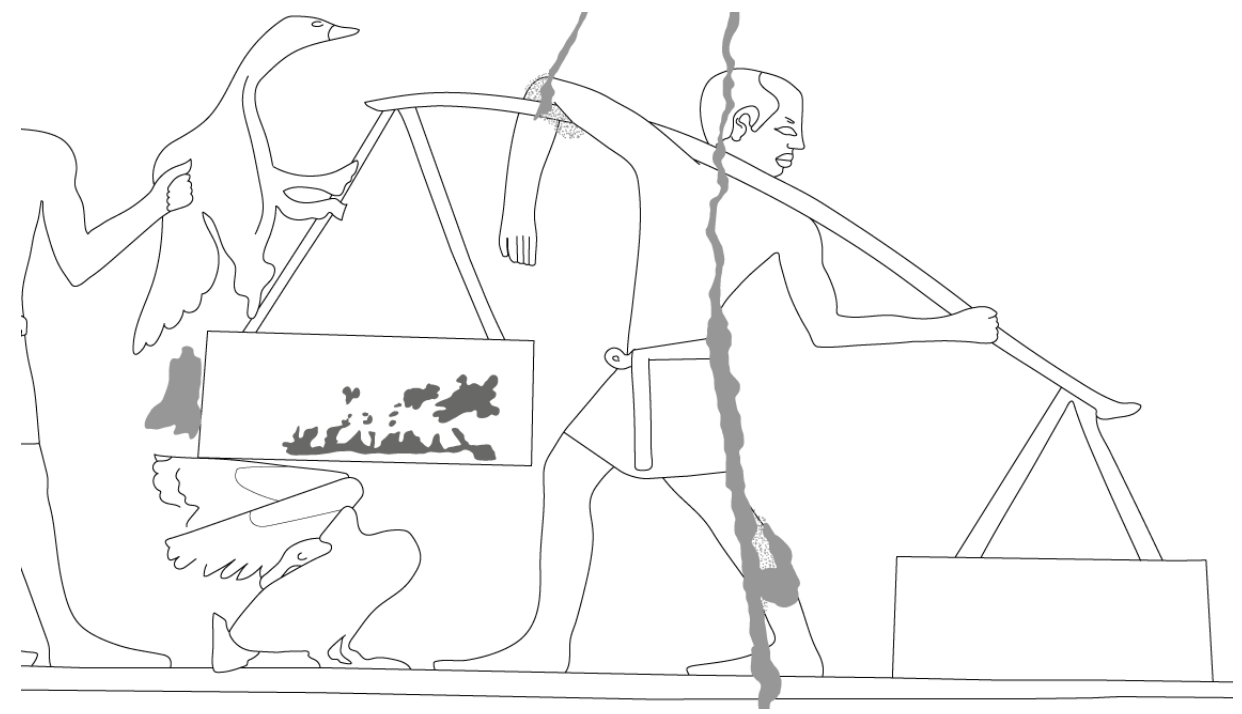

Fig. 1 : Porteur de palanche figurant dans le mastaba de Néfer et Kahai

Si l'on peut, dans un premier temps, penser à une posture de type BM-PP, on reste interpelé par un problème : comment le porteur a-t-il disposé sa palanche ?

Il est en effet physiquement impossible pour lui de faire passer en même temps la palanche devant son cou, sous son pli du coude et devant son bras. Si l'on met de côté l'hypothèse un peu facile d'une erreur du dessinateur, il faut pour expliquer cette anomalie considérer que cet homme est vu de dos ou au moins de trois quarts dos. Si cette lecture n'est pas évidente de prime abord, c'est parce que notre vision est perturbée par les jambes et le pagne qui ne sont pas dessinés de la manière attendue pour un œil habitué à la perspective. Toutefois, si l'on masque les jambes du personnage, l'intention du sculpteur est plus claire (fig. 2).

\footnotetext{
${ }^{3}$ On connaît pour ce type trois autres exemples, malheureusement lacunaires. Outre celui provenant du mastaba FS 3080 dont il sera question plus tard dans l'article, on retrouve une occurrence dans la tombe de Nebemakhet, S. HASSAN, Excavations at Gîza IV: 1932-1933, Le Caire 1943, pp. 135-136, fig. 77, pl. XXXVII et un autre dans le mastaba de Kaiemhéset, A. MCFARLANE, Mastabas at Saqqara: Kaiemheset, Kaipunesut, Kaiemsenu, Sehetepu and Others, (ACE Reports 20), Oxford 2003, p. 32, pl. 43.
} 


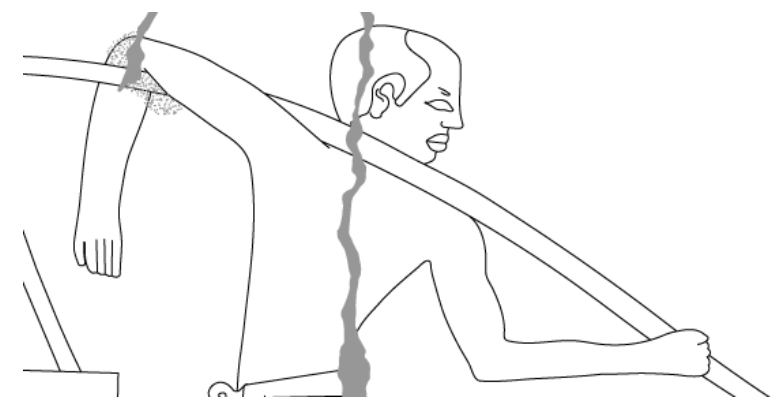

Fig. 2 : Détail de la scène

Une fois établi le fait que ce porteur est vu de dos, force est de constater que l'exécution de ce bas-relief est plutôt réussie et comparable aux standards et canons esthétiques de l'art tels qu'on le conçoit dans le monde occidental. Proposer une comparaison avec la statue figurant ci-dessous nous permet donc d'en apprécier les qualités plastiques tout en nous aidant à mieux visualiser comment le dessinateur percevait son œuvre en trois dimensions (fig. 3).

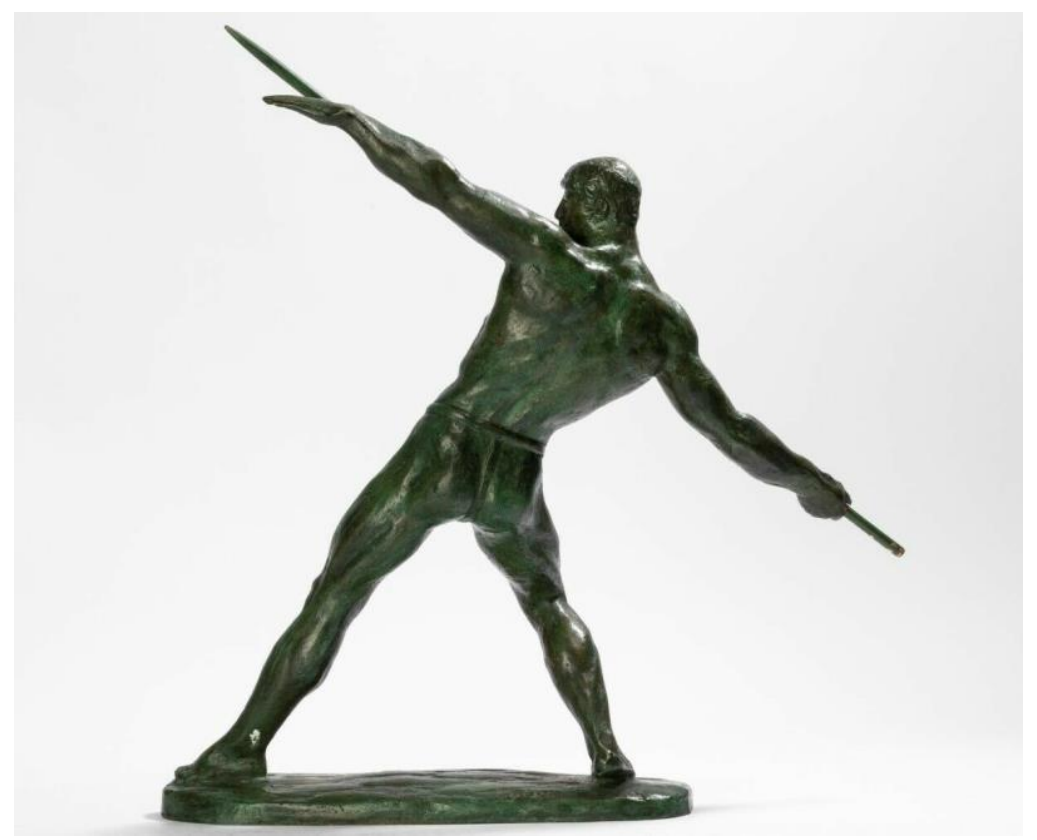

Fig. 3 : J. C. Guero, Lanceur de javelot. Statue art déco en bronze, circa 1930-1940 (C) Henri Hayat) 
Si les objectifs des deux personnages ne sont pas les mêmes - le lanceur s'apprête à se donner de l'élan pour projeter son javelot, ce qui n'est évidemment pas le cas du porteur et de sa palanche -, comparer ces deux œuvres nous permet de préciser les intentions de l'artiste égyptien.

S'agissant des bustes (fig. 4) on remarquera un traitement identique dans l'arcature du dos voire dans celui des épaules, les personnages étant tout deux légèrement penchés en arrière, le dos arqué et le bras gauche levé.
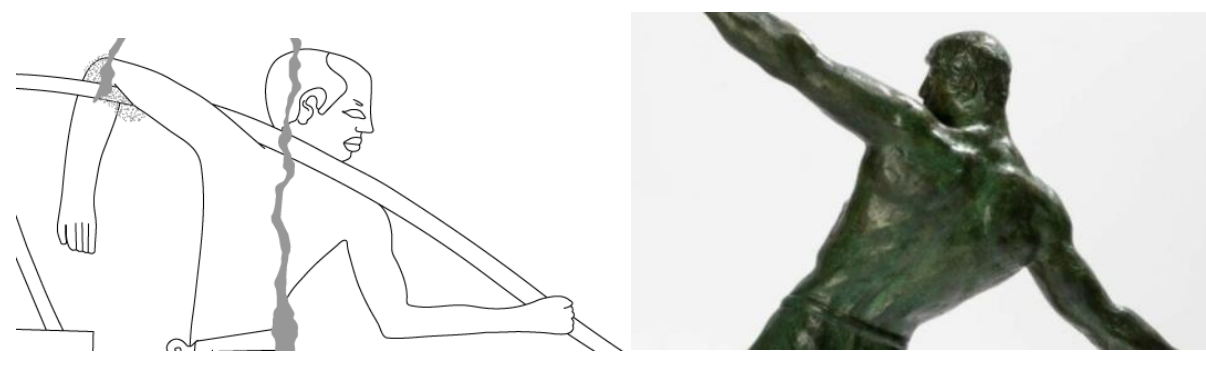

Fig. 4 : Comparaison des bustes

S'agissant des jambes (fig. 5), et bien qu'il existe des différences, là aussi la comparaison nous permet de préciser ce qu'a voulu représenter l'artiste égyptien. Alors que le lanceur s'appuie sur sa jambe droite qui est fléchie pour pouvoir ensuite, lors de sa détente, se donner plus de force pour propulser son javelot, le porteur est lui appuyé sur ses deux jambes dans une position de repos. Toutefois, malgré ces différences dans les intentions des personnages, leurs jambes droites sont en fait représentées de la même manière. Mais l'obligation pour l'artiste égyptien de devoir figurer le profil du pied droit a brouillé la lecture.
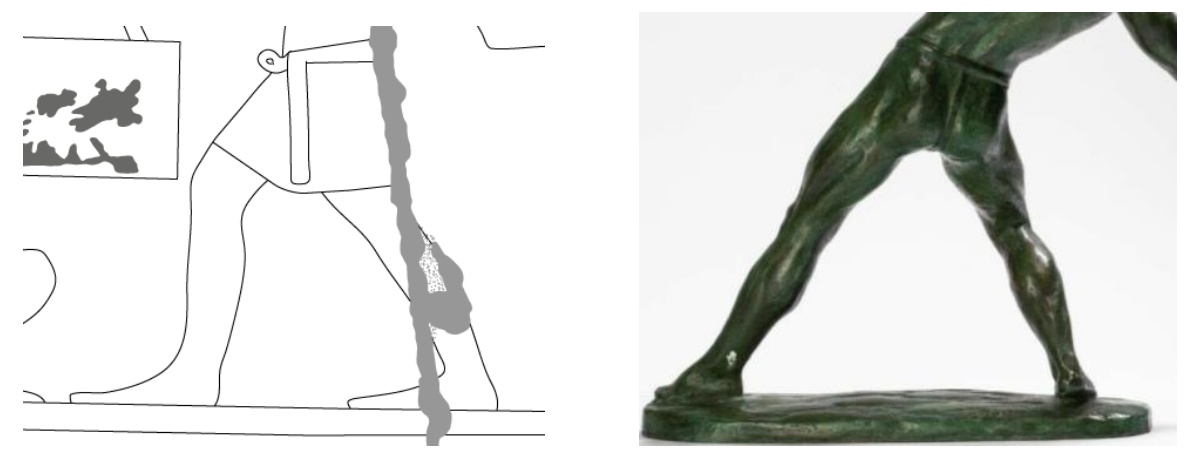

Fig. 5 : Comparaison des jambes 


\subsection{Restitution de la vue frontale}

Il est assez facilement possible d'imaginer la vue frontale de ce bas-relief (fig. 6).

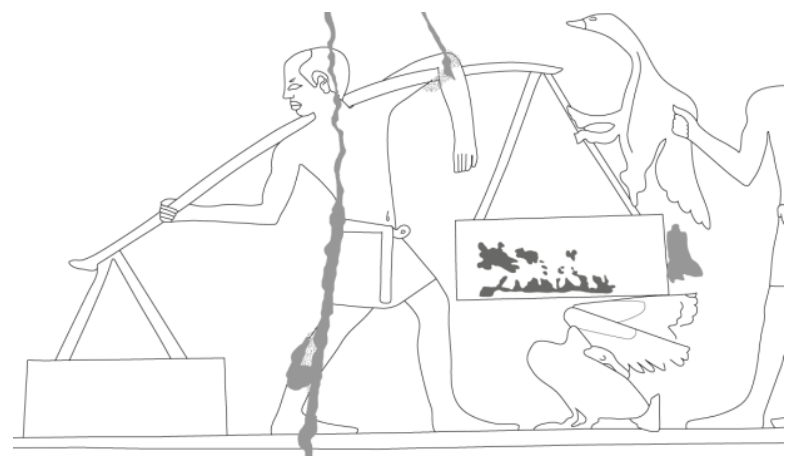

Fig. 6 : Le porteur, vue de face (création de l'auteur)

La figuration obtenue est dès lors similaire à ce que l'on peut observer ailleurs en Égypte, comme par exemple dans la tombe de Kaemnéféret (fig. 7 a). On notera également que la posture du lanceur de javelot de la statue de C. Guero est en vue frontale (fig. 7 b) semblable à celle du porteur de palanche du mastaba de Néfer et Kahai (fig. 6).
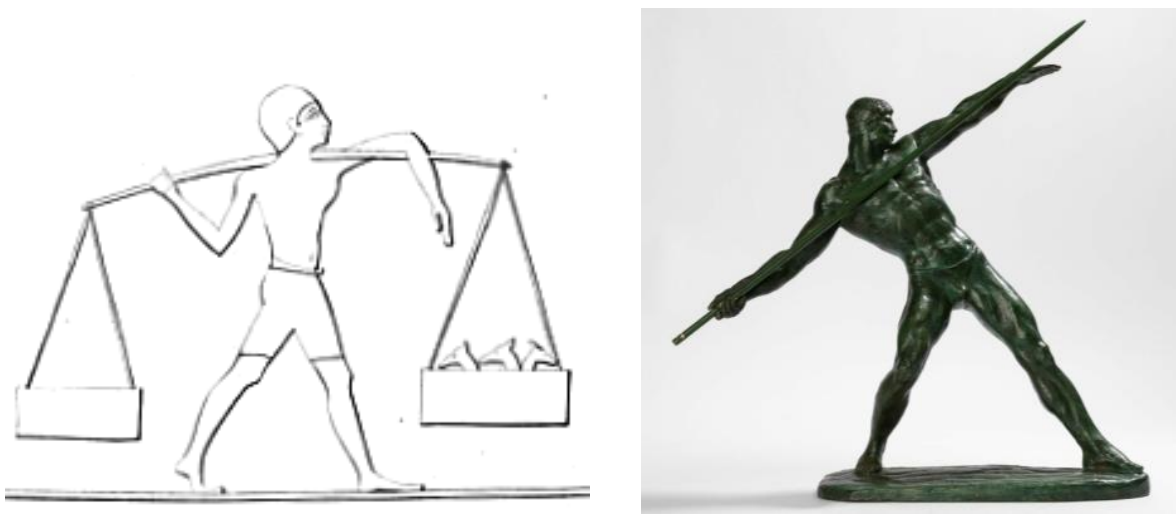

Fig. 7 : (a) Porteur dans la tombe de Kaemnéféret (LG 63) ;

(b) C. Guero, Lanceur de javelot (C Henri Hayat)

La création de cette vue frontale permet également de préciser les intentions de l'artiste dans l'exécution des jambes du porteur qui sont ici bien moins surprenantes, l'ensemble du dessin répondant aux normes habituelles. Pourtant elles ne sont que la vue en miroir des jambes du bas-relief. On est donc amené à 
se demander si l'artiste avait préalablement produit en esquisse une vue frontale similaire à celle proposée ici avant la réalisation de la vue dorsale. Dans ce cas il se serait contenté de reproduire les jambes sans les retoucher.

Enfin, cette restitution de vue frontale est également à comparer avec celle d'un porteur de palanche figurant dans le mastaba FS 3080.

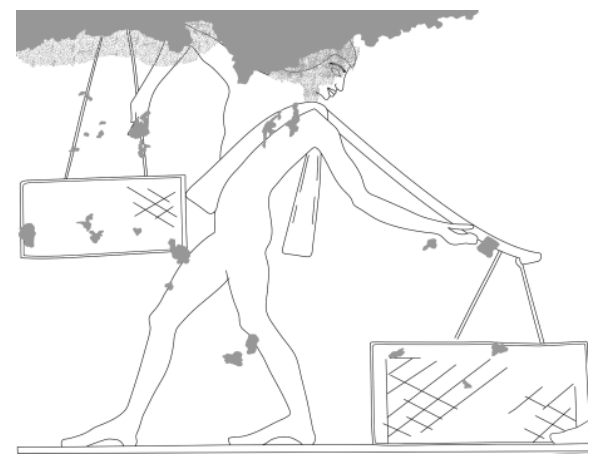

Fig. 8 : Porteur dans le mastaba FS 3080

Si la seule photographie ${ }^{4}$ à notre disposition ne permet pas de discerner avec certitude la position de la palanche et du bras du porteur, il semble toutefois que ce dernier soit bien vu de face. Son bras ne paraît pas posé sur la palanche mais appuyé contre, dans une position semblable à celles visibles dans le mastaba d'Ânkhmahor (MM-Pb dans le tableau) ou dans la tombe de Hési (MM-Cb dans le tableau).

\subsection{Un mouvement en cours}

Que ce personnage soit représenté de dos n'est pas le seul élément remarquable de ce bas-relief. Dans cette scène l'artiste a tenu également à montrer un mouvement en cours. Le porteur s'apprête en effet à se retourner pour basculer la palanche de l'autre côté et présenter la caisse vide à l'homme à côté de lui. Ce mouvement, à venir ou en cours, est signalé par le regard ${ }^{5}$ que porte le personnage sur la caisse située à sa droite (fig. 9 a). Comme pour la vue frontale, il est là aussi assez aisé d'imaginer ce à quoi aurait pu ressembler l'étape suivante (fig. 9 b).

${ }^{4}$ W.S. SMITH, A History of Egyptian Sculpture and Painting in the Old Kingdom, Boston 1949, pl. 51 [a].

${ }^{5}$ Aspect déjà remarqué par A.M. Moussa et H. AltenMüLLER, op. cit., p. 23. 

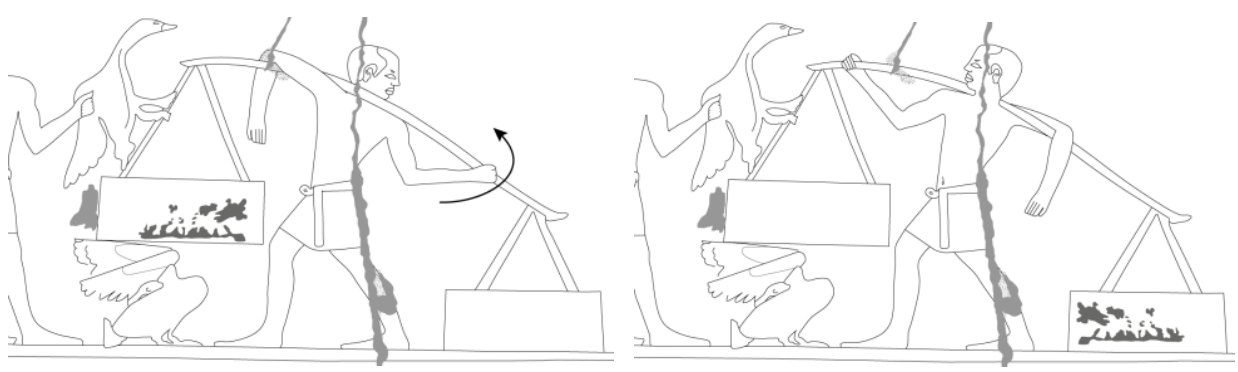

Fig. 9 : (a) Mouvement que s'apprête à faire le porteur.

(b) Posture finale du porteur (création de l'auteur)

Ces différents éléments permettent d'établir que cet artiste avait des facilités à se projeter dans un espace en trois dimensions - la vue frontale étant aisément restituable - voire en quatre dimensions - la vue finale étant là aussi restituable. Ils témoignent également que l'artiste avait réfléchi en amont aux objectifs et effets plastiques qu'il souhaitait atteindre.

Reste finalement à savoir pourquoi, en représentant un personnage de dos, l'artiste a tenu à figurer un mouvement ?

Dans la pensée égyptienne, telle que comprise aujourd'hui, les vues dorsales peuvent signifier deux choses : le désordre ${ }^{6}$ ou le mouvement ${ }^{7}$. On pourrait ajouter que c'est justement parce qu'elles marquent un désordre qu'elles créent un mouvement: il s'agit en effet pour le spectateur de réparer mentalement cette anormalité, de corriger cette transgression au canon ${ }^{8}$. En forçant le spectateur à imaginer la vue frontale (fig. 6) ou la vue finale (fig. 9 b) de la scène, l'artiste crée un « thaumatrope ${ }^{9}$ mental »: l'œil voit une première image que le cerveau corrige dans une seconde image, enfermant ainsi ce porteur dans un perpétuel mouvement de remplissage de sa palanche. Si l'on part sur cette hypothèse de lecture, le

${ }^{6}$ D. FArout, «La frontalité contrariée dans l'iconographie égyptienne », (Pallas [En ligne] 105), 2017, consulté le 9 juin 2021, § 26.

${ }^{7}$ L'utilisation des vues dorsales pour signifier le mouvement a depuis longtemps été identifiée par de nombreux chercheurs. Citons par exemple, s'agissant de l'Ancien Empire, S.A. GoudSMIT, «The Back View of Human Figures in Ancient Egyptian Art» (JNES 40.1), 1981, pp. 43-45. De nombreux autres auteurs qui ont travaillé sur la question sont également cités dans A. EL-SHAHAWY, Recherche sur la décoration des tombes thébaines du Nouvel Empire: Originalités iconographiques et innovations (IBAES XIII), Londres 2010, n. 993, 994, 1062.

${ }^{8}$ Correction qui, si j'y suis parvenu après moult réflexions, devait être à mon avis automatique chez un Égyptien.

${ }^{9}$ Le thaumatrope, du grec thauma, prodige et tropion, tourner, est un jouet d'optique réinventé $\mathrm{au} \mathrm{XIX}{ }^{\mathrm{e}}$ siècle. Le plus connu est sans aucun doute celui figurant sur une face un oiseau et sur l'autre une cage vide qui mis en mouvement donne à voir un oiseau en cage. 
« raté » dans la représentation des jambes n'en est donc peut-être plus un mais participe à créer cette illusion : les jambes étant vues de face quand le buste est vu de dos, cette contradiction oblige le spectateur à envisager les deux possibilités et à opter pour l'une ou l'autre tout en le laissant dans l'incapacité de le faire. Pour étayer cette hypothèse on ajoutera que ce « thaumatrope mental » est renforcé par les autres personnages du registre qui sont vus de profil - offrant ainsi au spectateur un aperçu de l'une des étapes intermédiaires du retournement du porteur de palanche - et que le personnage à côté du porteur est lui, vu de face offrant ainsi au spectateur un aperçu de la vue finale.

Plusieurs raisons, qui ne s'excluent pas nécessairement les unes et les autres, peuvent expliquer la présence de ce «thaumatrope mental». Ainsi l'on peut penser à un désir d'innovation de la part de l'artiste, cet exemple n'étant pas le seul à figurer dans cette tombe ${ }^{10}$. Mais plus qu'une simple envie de bousculer les codes, il est envisageable qu'il y ait de la part de l'artiste et/ou du commanditaire une envie de renforcer l'aspect performatif des scènes. En y ajoutant du mouvement, les actes représentés n'en sont que plus efficients. Et en inscrivant cette scène dans un mouvement perpétuel, le commanditaire s'assure un approvisionnement pour l'éternité.

\section{Conclusion}

Depuis que les canons de l'art égyptien antique ont été établis par Heinrich Schäfer puis Emma Brunner-Traut ${ }^{11}$ à sa suite, on a pu constater qu'il existe de nombreux exemples de transgressions de ces normes. Se nichant souvent dans des détails tels que la position d'une main, d'un bras, d'un pied, etc., nombreuses sont les indications permettant de donner du relief, de la profondeur, du mouvement aux scènes. Ces éléments, qui semblent cependant n'être le fait que d'une partie des artistes - dont la portion reste à établir - questionnent sur les raisons ayant amené à ces innovations. Volonté d'apporter du réalisme et de la vitalité ou simple envie transgressive, ces exemples sont-ils l'exception ou font-ils partie de la règle ?

\footnotetext{
${ }^{10}$ A. BRÉMONT, « 'Aspectivité' ou plutôt 'multispective' ? Les leçons du paradoxe de la chèvre », Nehet 4 (2016), pp. 25-44.

${ }^{11}$ H. SCHÄFER, Principles of Egyptian art ; edited with an epilogue by Emma Brunner-Traut ; translated and edited by John Baines with a foreword by E. H. Gombrich, Oxford 2002.
} 
Sources des documents

Doc 1 : H.T. MoHR, The Mastaba of Hetep-her-ahkti. Study on an Egyptian Tomb Chapel in the Museum of Antiquities, Leyde 1943, p. 58, fig. 28.

Doc 2 : A. M. Moussa, H. AltenMÜLleR, The Tomb of Nefer and Ka-Hay (ArchVer 5), Mayence 1971, pp. 21-23, pl. 5 ; M. LASHIEN, The Chapel of Kahai and his Family (ACE Reports 33), Oxford 2013, pp. 30-31, pl. 16, 81-82.

Doc 3 : K. R. LePsIUS, Denkmäler aus Aegypten und Aethiopien II, Band III, Berlin 1849-1859, pl. 54 ; K. R. WeEKS, Mastabas of Cemetery G 6000 (Giza Mastabas 5), Boston 1994, p. 52, fig. 42, pl. 28.

Doc 4 : N. Kanawati, The Rock Tombs of El-Hawawish: The Cemetery of Akhmim, I, Sydney 1980, p. 27, pl. 10 [b], fig. 15.

Doc 5 : N. de G. DAVIES, F. L. GRIFFITH, The Mastaba of Ptahhetep and Akhethetep at Saqqarah. Part I, the Chapel of Ptahhetep and the Hieroglyphs (ASEg. 8), Londres 1900, p. 10 et pl. XXI, XXV.

Doc 6 : Pr. Duell, The Mastaba of Mereruka. Part I. Chambers A 1-10. Plates 1-103, (OIP 31), Chicago 1938, pl. 10, 13 ; N. Kanawati, A. WoOdS, S. SHafiK, E. AleXAKIS, Mereruka and his Family, Part III: 1, The Tomb of Mereruka (ACE Reports 29), Warminster 2010, pl. 14, 67 et 68.

Doc 7 : H. WILD, Le Tombeau de Ti, Fasc. III, La chapelle (deuxième partie) (MIFAO 65), Le Caire 1966, pl. CLXVI.

Doc 8: P. MunRO, Der Unas-Friedhof Nord-West. Topographisch-historische Einleitung. Das Doppelgrab der Königinnen Nebet und Khenut, Mayence 1993, p. 139, pl. 42.

Doc 9: N. Kanawati, The Rock Tombs of El-Hawawish: The Cemetery of Akhmim, VIII, Sydney 1988, p. 12, pl. 2 [a], 8 [a], fig. 3 [b].

Doc 10 :N. Kanawat, The Rock Tombs of El-Hawawish : The Cemetery of Akhmim, III, Sydney 1982, pp. 40-41, fig. 27, pl. 10.

Doc 11 :N. KanaWATI, A. HASSAN, The Teti Cemetery at Saqqara, Volume II : The Tomb of Ankhmahor (ACE Reports 9), Warminster 1997, pp. 37-38, pl. 7 [b] et 41.

Doc 12 :S. D'Auria, P. Lacovara, C. H. Roehrig, Mummies \& Magic : the Funerary Arts of Ancient Egypt, Boston 1988, pp. 83-87.

Doc 13 :N. Kanawati, M. ABDER-RAZIQ, The Teti Cemetery at Saqqara, Volume V : The Tomb of Hesi (ACE Reports 13), Warminster 1999, pp. 29-32, pl. 55.

Doc 14 : N. Kanawati, A. Hassan, The Teti Cemetery at Saqqara, Volume II : The Tomb of Ankhmahor (ACE Reports 9), Warminster 1997, pp. 37-38, pl. 7 [b] et 41.

Doc 15 :N. Kanawati, M. ABdeR-RAZIQ, The Teti Cemetery at Saqqara, Volume V : The Tomb of Hesi (ACE Reports 13), Warminster 1999, pp. 29-32, pl. 55.

Doc 16 :W. S. SMITH, A History of Egyptian Sculpture and Painting in the Old Kingdom, Boston 1949, pl. 51 [a]. 
Doc 17 :K. R. LePsIUS, Denkmäler aus Aegypten und Aethiopien II, Band III, Berlin, 1849-1859, pl. 9 [b].

Doc 18 :Pr. Duell, The Mastaba of Mereruka. Part I. Chambers A 1-10. Plates 1-103 (OIP 31), Chicago 1938, pl. 20-21; N. Kanawati, A. Woods, S. ShafiK, E. ALEXAKIs, Mereruka and his Family, Part III: 1, The Tomb of Mereruka (ACE Reports 29), Warminster 2010, pl. 17, 69 et 70. 